\title{
Colloque « Kubrick, les films, les musiques »
}

Université d'Évry, 25-26 mars 2011

\section{Serge Dambrine}

\section{(2) OpenEdition}

\section{Journals}

Édition électronique

URL : https://journals.openedition.org/transatlantica/5417

DOI : 10.4000/transatlantica.5417

ISSN : $1765-2766$

Éditeur

Association française d'Etudes Américaines (AFEA)

\section{Référence électronique}

Serge Dambrine, «Colloque «Kubrick, les films, les musiques»», Transatlantica [En ligne], 2 | 2011, mis en ligne le 11 juin 2012, consulté le 10 février 2023. URL : http://journals.openedition.org/ transatlantica/5417 ; DOI : https://doi.org/10.4000/transatlantica.5417

Ce document a été généré automatiquement le 10 février 2023.

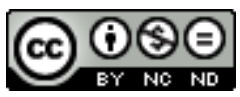

Creative Commons - Attribution - Pas d'Utilisation Commerciale - Pas de Modification 4.0 International - CC BY-NC-ND 4.0

https://creativecommons.org/licenses/by-nc-nd/4.0/ 


\section{Colloque « Kubrick, les films, les musiques »}

Université d'Évry, 25-26 mars 2011

\section{Serge Dambrine}

1 SCRIPT ${ }^{1}$, centre de recherche en émergence à l'Université d'Évry, conçu comme une plateforme des arts engagés très contemporains, organisait au printemps sa première manifestation scientifique. Alors que la Cinémathèque Française inaugurait l'exposition "Stanley Kubrick», américanistes, filmologues, musicologues et praticiens de la communication se réunissaient sur le campus d'Évry pour examiner ensemble l'œuvre du grand cinéaste mort en 1999.

2 Photographe et joueur d'échecs, féru de lecture autant que d'image et de musique, créateur reconnu pour son talent de visionnaire et communicateur soucieux du rapport au spectateur, Stanley Kubrick a suscité l'intérêt de chercheurs d'horizons divers. C'est pourquoi Brigitte Gauthier, qui ouvrait le colloque en qualité de responsable du nouveau laboratoire et de la nouvelle UFR de Langues, Arts et Musique à Évry, s'est réjouie d'en aborder l'œuvre avec ses collègues et invités, dans une optique délibérément multidisciplinaire.

3 Nous avons regroupé les communications autour des trois principales sources créatives dont elles se font l'écho : le motif et la figure expressive, l'écrit (le roman transposé) et l'élément sonore (l'œuvre musicale convoquée).

\section{Du motif (ou de la figure) au film}

Dans sa communication inaugurale, "Kubrick: cinéaste compositeur", Brigitte Gauthier (études anglophones et filmologie, Université d'Évry) s'est intéressée à la fonction hypnotique de l'image et du son chez Kubrick, et en particulier dans The Shining (1980). Pour elle, les grands thèmes du cinéaste - pouvoir et folie - justifient une dramaturgie de l'hypnose, capable d'emmener le spectateur à la charnière de la normalité et de la déviance - frontière sur laquelle se meuvent les personnages. Reléguant le texte à un rôle secondaire, musique et image se répondent en écho, 
composées avec une précision implacable et la plus grande beauté formelle. Enchaînées souvent par "simple " association d'idées jusqu'à une extrême tension, elles captent le spectateur. Neutralisé, celui-ci glisse dans la perception des personnages et revêt le Kubrick's stare, regard hypnotique et regard hypnotisé. Ainsi le cinéaste interroge, selon les mots de Brigitte Gauthier, la «possibilité de filmer [...] les confins filmiques du rationnel et de l'irrationnel ».

Intervenant sur « les rapports homme-machine chez Stanley Kubrick », Christian Romain (auteur et consultant en marketing, Paris) a examiné quatre films dans lesquels le cinéaste met en scène, plus ou moins ouvertement, une certaine relation de l'homme à la machine : Dr. Strangelove (1964), 2001 : A Space Odyssey (1968), The Shining et Full Metal Jacket (1987). Selon l'intervenant, l'enjeu du pouvoir conféré aux machines et le péril de l'identification de l'homme à la machine sont deux thèmes récurrents de ces films qui, pourtant, en cachent un troisième: la circularité des destins humains, dont la répétition, notamment mécanique, tient lieu de métaphore. Cette boucle dynamique est d'ailleurs un motif général, au-delà des quatre films cités. De fait, a précisé Christian Romain, Spartacus (1960) mis à part (Kubrick l'a renié), seul 2001, dont la circularité s'ouvre en spirale par une intervention extérieure de nature mystérieuse, échappe à ce retour à l'état antérieur et à la figure de l'homme comme marionnette bégayante.

Intervenant sur « Kubrick et la voix off : analyse filmométrique de l'Ultime Razzia », Javier Sanchez (linguistique informatique, études hispaniques et traduction spécialisée, Université d'Évry) a présenté son analyse lexicométrique des interventions du narrateur principal omniscient dans The Killing (1956). Si les quatre séquences syntaxiquement dominantes (introduction du discours direct et de l'action dramatique), notamment placées au début du film, permettent de dévoiler l'intimité des protagonistes, l'intervenant a révélé que les interventions off de nature syntaxiquement dépendante (introduction des personnages, pointage des paramètres spatio-temporels de la séquence) sont de loin les plus nombreuses (il en dénombre 26). Économiques en formes (mots) comme en temps, elles livrent, selon Javier Sanchez, les clés d'une «narration accélérée et déconstruite » sur le motif du puzzle. Ce faisant, la voix off ponctue d'une manière entêtée le déroulement sous tension d'un plan que Kubrick a littéralement mis en pièces.

7 Puis Alain Cohen (littérature comparée et cinéma, University of California, San Diego), dans " The Killing dans l'œuvre de Kubrick. Rythme et silence de la voix off ", s'est livré à une étude structurale du même film. Rappelant les traits communs du heist genre (tension, ponctualité, échec final du braquage, arrestation et mort du protagoniste rebelle), il a montré le rôle formel de la voice over, gardien insistant du bon déroulement du plan et contrepoint de la voix assurée du héros, Johnny Clay. Or, lorsque Johnny constate, la voix éteinte, l'échec de son entreprise, la voix off se tait. Reprenant le système dramatique du film, Alain Cohen a proposé plusieurs interprétations de ce mutisme. Toutes prêtent à la voix off les traits du macguffin hitchcockien - ce motif moteur et récurrent du récit qui, devenu inutile, disparaît subitement. Chacune, en revanche, attribue à la voix off une fonction psychologique distincte, liée à la personnalité de Johnny, braqueur incurable - et autre figure de la répétition.

8 Abordant le motif de l'illusion dans "2001, l'avènement des effets visuels. De la technique à l'esthétique kubrickienne", Réjane Vallée (sociologie et audiovisuel, Université d'Évry) a rappelé l'apport de Kubrick au développement des effets spéciaux. Si le principe technique (effacer visuellement la cause de l'effet, porter l'attention du 
spectateur sur les éléments crédibles de l'image) est en soi matière à défi, l'intervenante a montré comment Kubrick s'inscrit dans une recherche esthétique dont les effets sont une partie essentielle, non pas seulement une condition formelle. Kubrick conçoit 2001 comme une expérience visuelle; il innove donc, employant le slit scan, l'alliance du maquillage et de l'animatronique, et la front projection. Ainsi le cinéma passe d'une économie de l'effet spécial - accessoire de la narration (que Réjane Vallée relie à Georges Méliès) - à celle de l'« effet film », préparant l'avènement des effets visuels dans les années 1980 (qu'elle relie à George Lucas).

\section{De l'écrit au film (et vice-versa)}

Dans une étude consacrée au dernier film du cinéaste, « De la Vienne de Schnitzler au New York de Kubrick : Eyes Wide Shut ", Christine Bonneville (langue et littérature germaniques, Université d'Évry) a précisé les proximités de Schnitzler et Kubrick pour mieux comprendre la fidélité du film (1999) à la Traumnovelle (1926), dont il est tiré. Thématiquement, juge l'intervenante, ce sont les relations familiales comme objet d'écriture, et l'écriture même comme terrain d'investigation psychologique qui rapprochent les deux créateurs. Formellement, ce sont la distance ironique, le motif du rêve et, plus généralement, une façon d'appréhender l'écriture filmique, en particulier dans la rigueur structurelle, l'utilisation de la musique et des dialogues. Selon Christine Bonneville, Kubrick trouve aussi dans le dénouement de la nouvelle l'héritage humaniste du médecin viennois qui, fin connaisseur de la psychanalyse et défenseur du concept de semi-conscience (Mittelbewusstsein), tentait de restituer à l'homme une part de responsabilité dans son comportement.

Étude génétique d'une autre adaptation, la communication de Jocelyn Dupont (littérature et cinéma anglophone, Université de Perpignan), "Écritures de Barry Lyndon » (1975), concernait le script de pré-production du film, dont le scénario est le seul que le cinéaste ait écrit lui-même. De l'analyse de ce script, conservé au British Film Institute, l'intervenant conclut que le projet initial de Kubrick est fidèle au roman de Thackeray, dans son organisation diégétique comme dans le choix des péripéties; Kubrick l'étoffe toutefois, insérant des séquences qui confèrent au projet de film les traits d'un récit de cape et d'épée teinté de farce. Rien n'annonce la recherche esthétique du produit final : c'est donc dans un second lieu, celui de la composition filmique, que Kubrick délaisse radicalement le texte original pour en faire le chefd'œuvre visuel que l'on connaît - ce qui enrichit, a conclu Jocelyn Dupont, notre appréhension des collaborations (difficiles) du cinéaste avec ses scénaristes.

Dans "Les Lolita de Nabokov et Kubrick : traductions de la censure", Stephanie Genty (littérature américaine et langues de spécialité, Université d'Évry) s'est penchée sur l'adaptation du roman de Nabokov. Kubrick, qui en acquiert les droits à un moment crucial dans sa carrière, doit recevoir l'aval de la Production Code Administration tout en trouvant un financement qui lui laisse les mains libres. Selon l'intervenante, l'histoire du scénario est donc celle d'un défi : détourner un récit pour contourner les censeurs, censurant incidemment l'écrivain engagé comme scénariste. Au prisme de la traductologie, Stephanie Genty a démonté les omissions, transformations et déplacements qui permirent à Kubrick de mettre en image un roman perçu comme indécent, non sans critiquer le puritanisme de son époque : c'est bien pour elle le 
produit d'une traduction «oblique » qui sortit sur les écrans en 1962, permettant au jeune réalisateur de confirmer sa stature de cinéaste accompli.

Sur le même film, l'étude d'Olivier Caïra (sociologie et cinéma, IUT d'Évry), « Les rôles masculins dans Lolita: du roman à l'écran", s'intéressait aux personnages de Humbert et de Quilty. Observant combien Kubrick dépouille le statut de narrateur réflexif du Humbert nabokovien, sacrifiant l'ironie et la polyphonie qui fondent le comique du roman à une forme d'autocensure, l'intervenant a mis en évidence le mécanisme de vases communicants par lequel se modèlent les deux personnages masculins dans le film. En un comique de situation monté de toutes pièces, Humbert Humbert devient le jouet d'un amour impossible, la proie d'un puissant conflit intérieur et la victime d'un choc de cultures; inversement, Clare Quilty décuple ses talents de manipulateur et déploie une perversion tapageuse et multiforme, traits originels du premier. Pour Olivier Caïra, cette mutation, amorcée par Nabokov scénariste, construit donc les deux personnages par opposition et rend ainsi le film acceptable aux censeurs.

Enfin, dans "Genres, normes et adaptations dans la filmographie de Kubrick ", Sabrina Baldo (langue et littérature anglaise, traduction spécialisée, Université d'Évry) s'est penchée sur la question des sous-titres français dans les films de Kubrick. Rappelant que l'adaptation (sous-titrage) se pratique dans une tension constante entre l'impératif de réduction du message-source et le risque d'omettre un élément essentiel, l'intervenante a relevé l'importance qu'elle revêtait pour le cinéaste américain : celui-ci exigeait de contrôler systématiquement les sous-titres. Quoique connu pour son utilisation narrative de l'image et du son, Kubrick met en effet le traducteur à l'épreuve : logorrhées de personnages à l'accent marqué ou au vocabulaire obscène (Quilty dans Lolita, le sergent dans Full Metal Jacket), dialogues empreints d'une fausse banalité (Hal dans 2001, personnages principaux d'Eyes Wide Shut) et jeux de mots intraduisibles (The Shining), figurent, pour Sabrina Baldo, parmi les difficultés les plus remarquables.

\section{De la musique au film (et vice-versa)}

Dans sa communication « Le film comme partition. Musique et narration dans les films classiques de Kubrick ", Giovanni Robbiano (scénariste, réalisateur, producteur et enseignant en cinéma, Gênes) postule qu'un film est une combinaison de plans, qu'un morceau de musique est une combinaison de notes, et que les rapports entre les plans et entre les notes donnent tout leur sens à ces combinaisons comme à l'ensemble qu'elles constituent. Sur ces bases, l'intervenant a tenté d'établir un parallèle entre cinéma et musique. S'ils ne sont pas conçus pour la lecture, ces deux modes d'expression procèdent bien d'une écriture et requièrent une structure. Kubrick, qui excellait à renouveler le langage filmique, fait de la musique un outil narratif et la manie avec son ironie de réalisateur engagé. Appuyant son analyse sur les musiques de 2001 et Dr Strangelove, Giovanni Robbiano a ainsi soutenu qu'avec Kubrick, la musique a pris une nouvelle importance dans le cinéma, à l'égal de la photographie et du script.

15 L'intervention de Gilles Ménégaldo (littérature américaine et cinéma, Université de Poitiers), intitulée «Musique et conventions génériques dans Lolita et The Shining de Stanley Kubrick », présente deux postures musicales distinctes dans « le jeu plus ou moins subversif et parodique » que le cinéaste pratique avec les conventions de genre. 
Dans Lolita, film clairement hybride comme le roman de Nabokov, chaque motif musical se lie à un personnage ou à une situation, à la manière d'un leitmotiv souvent ironique. Dans The Shining, qui ne relève certes pas seulement du fantastique ou de l'horreur, Kubrick rompt avec la tradition de ces genres : à part deux compositions originales (et de vieux airs dansants pour détendre l'ambiance et suggérer l'emprise du passé), il choisit des pièces de Bartok, Ligeti et Penderecki. Gilles Ménégaldo a analysé l'emploi que Kubrick fait de leurs œuvres (et notamment leurs effets obsessifs de répétition et d'enchevêtrement) pour susciter à sa manière l'angoisse et la surprise. Wide Shut ", Chloé Huvet (doctorante en musicologie, École Normale Supérieure de Lyon) a examiné le rapport au regard qu'ont certaines des musiques récurrentes du dernier film de Kubrick. Selon l'intervenante, la seconde valse de la Jazz Suite de Chostakovitch contribue à distancier le spectateur dans les scènes qui l'introduisent ironiquement à l'intimité du couple de protagonistes. Les compositions de Jocelyn Pook associées au personnage de Bill, lorsqu'il fantasme l'adultère de sa femme puis pénètre dans le château de Somerton, nous conduisent au contraire avec lui hors de la réalité quotidienne. Enfin, la seconde pièce du recueil Musica Ricercata de Ligeti, qui place Bill sous tension puis sonne le glas et signifie sa chute, nous rejette dans un regard surplombant et hostile. Ainsi, pour Chloé Huvet, les œuvres choisies "assument pleinement la focalisation » adoptée par le réalisateur dans chacune des séquences du film.

Puis Damien Ehrhardt (musicologie, Université d'Évry), dans « Ludwig van : de Kagel à Kubrick ", a comparé le film Ludwig van (1969) de Maurizio Kagel et A Clockwork Orange (1971) de Stanley Kubrick, dont le personnage principal, Alex, est un aficionado obsessif du même "Ludwig van». Les deux films, apparus autour du bicentenaire de la naissance de Beethoven, découplent la figure mythique du compositeur de celle de Ludwig van, son alter ego quotidien. Kagel fait de celui-ci un musicien des extrêmes, spectre chahuté entre violence, décalage hasardeux et suspension temporelle ; Kubrick, accentuant l'intention de Burgess dans le roman qu'il transpose, en fait la figure obsessive de la Neuvième Symphonie, associée à l'expression de la violence. Ainsi, conclut Damien Ehrhardt, l'œuvre emblématique des valeurs de fraternité (bien qu'instrumentalisée par toutes les familles politiques du passé), trouve une personnification paradoxale en Ludwig van, figure radicalement démythifiée du compositeur.

Enfin, «La musique de Ligeti dans 2001: l'Odyssée de l'espace. Kubrick recompositeur ", communication de Roméo Agid (doctorant en musicologie, Université d'Évry) se fonde sur le postulat que l'inscription d'une musique existante dans un film constitue un détournement des intentions originales du compositeur, donc une recomposition. Par suite, et s'écartant d'une analyse fonctionnelle de la musique chez Kubrick, l'intervenant a relevé dans 2001 trois "aspects de l'écriture musicale du réalisateur » : la coïncidence du chant avec l'évolution de l'homme ("voix du mythe »), le monolithe noir conçu comme instrument de musique (pour lequel le cinéaste recompose l'œuvre de Ligeti) et le croisement des dimensions macroscopiques et microscopiques (« entrelacs musico-pictural subtil, en strates croisées »). Selon Roméo Agid, l'œuvre de Ligeti s'inscrit ainsi dans le théâtre cosmique de Kubrick. De fait, a-t-il défendu, 2001 s'apparente à une autre posture compositionnelle et formelle: celle de l'opéra - un opéra sans chanteur. 


\section{Pour aller plus loin}

19 Programme du colloque: http://www.univ-evry.fr/fr/l_universite/composantes/ ufr_langues_arts_et_musique/

colloque_international_kubrick_cineaste_compositeur.html.

À paraitre: Kubrick, les films, les musiques (2 volumes), Montpellier, l'Entretemps, coll.

«Les points dans les poches », 2012.

\section{NOTES}

1. Scénaristes, créateurs, réalisateurs, interprètes, performeurs et traducteurs.

INDEX

Thèmes : Actualité de la recherche

\section{AUTEUR}

\section{SERGE DAMBRINE}

Université d'Évry et Université Autonome de Barcelone 